\title{
ON THE COMPLETENESS OF LAMBERT FUNCTIONS*
}

\section{BY EINAR HILLE AND OTTO SZÁSZ}

1. Introduction. In the theory of Lambert series one encounters the following two sets of functions

$$
S_{1}: \frac{(1-t) t^{n}}{1-t^{n}}, \quad \text { and } \quad S_{2}: \frac{(1-t)^{2} t^{n}}{\left(1-t^{n}\right)^{2}}, \quad(n=1,2,3, \cdots) \text {. }
$$

Both sets evidently belong to the spaces $L_{p}(0,1), p \geqq 1$, and to $C[0,1]$, the space of functions continuous in the closed interval $[0,1]$, but nothing definite seems to be known about their closure properties in these various spaces. Wiener's work on the Lambert-Tauber theorem $\dagger$ suggests that they are complete in $L_{1}(0,1)$. On the other hand, a direct elementary proof of this fact would form a first step towards a simple proof of his theorem.

In the present note we shall prove completeness in $L_{p}(0,1)$, $p \geqq 1$, of sets of functions of the type

$$
S\left(\alpha, \beta, \lambda_{n}\right): \quad(1-t)^{\alpha} \sum_{m=1}^{\infty} m^{\beta} t^{m \lambda_{n}}, \quad(n=1,2,3, \cdots),
$$

under suitable restrictions on the parameters. It will turn out in particular that the sets $S_{1}$ and $S_{2}$ are complete in any Lebesgue space. The adjunction of $f_{\mathrm{v}}(t)=1$ to $S\left(\alpha, \beta, \lambda_{n}\right)$ leads to sets complete in $C[0,1]$, but the sets $S_{1}$ and $S_{2}$ turn out to be border-line cases in the discussion, and we are not able at present to prove that they can be made complete by this device.

The analysis is capable of very considerable extension. Thus we could replace the factor $(1-t)^{\alpha}$ by more general multipliers. A more interesting situation is encountered if we replace the coefficients $m^{\beta}$ by quantities $c_{m}$ such that $c_{1}=1,\left|c_{m}\right| \leqq M m^{\beta}$, $m>1$. Owing to the number theoretic features of the problem, which are introduced by the inversion formula of Möbius, this

* Presented to the Society, February 29, 1936.

$\dagger$ See Annals of Mathematics, (2), vol. 33 (1932), pp. 1-100, especially pp. 39-43, and his book, The Fourier Integral, 1933, pp. 112-124. 
slight change in the assumptions affects the analysis strikingly. This more general problem will be treated in a later paper.

2. Completeness in $L_{1}(0,1)$. We start with the case $p=1$, which leads to the following theorem.

THEOREM 1. Let

$$
k_{\beta}(w)=\sum_{m=1}^{\infty} m^{\beta} w^{m}, \quad(|w|<1) .
$$

Let $\alpha>\max (\beta,-1)$. Let $\left\{\lambda_{n}\right\}$ be a set of complex numbers such that (i) $\Re\left(\lambda_{n}\right) \geqq \delta>0$, and (ii) $\sum_{n=1}^{\infty} \Re\left(1 / \lambda_{n}\right)=+\infty$. Then the set

$$
S\left(\alpha, \beta, \lambda_{n}\right): \quad(1-t)^{\alpha} k_{\beta}\left(t^{\lambda_{n}}\right), \quad(n=1,2,3, \cdots),
$$

is complete in $L_{1}(0,1)$.

Proof. A set of functions $f_{n}(t) \epsilon L_{1}(0,1)$ is complete in this space if and only if the only bounded measurable function $g(t)$ satisfying

$$
\int_{0}^{1} f_{n}(t) g(t) d t=0, \quad(n=1,2,3, \cdots),
$$

is $g(t) \sim 0$. Let us apply this criterion to the set $S\left(\alpha, \beta, \lambda_{n}\right)$. Let $z=x+i y$, and form the function

$$
F(z)=\int_{0}^{1} k_{\beta}\left(t^{z}\right)(1-t)^{\alpha} g(t) d t
$$

for $x>0$. If $|g(t)| \leqq g$,

$$
|F(z)| \leqq g \int_{0}^{1} k_{\beta}\left(t^{x}\right)(1-t)^{\alpha} d t .
$$

If $\beta<-1, k_{\beta}(u)$ is bounded for $0 \leqq u \leqq 1$. Since $\alpha>-1,|F(z)|$ is clearly bounded for $x \geqq 0$. If $\beta>-1$, we can find a constant $M_{\beta}$ such that $k_{\beta}(u) \leqq M_{\beta}(1-u)^{-\beta-1}$. Hence

$$
\begin{aligned}
|F(z)| & \leqq g M_{\beta} \int_{0}^{1}\left(1-t^{x}\right)^{-\beta-1}(1-t)^{\alpha} d t \\
& =g M_{\beta} \Gamma(\alpha+1) \sum_{m=0}^{\infty}(-1)^{m}\left(\begin{array}{c}
-\beta-1 \\
m
\end{array}\right) \frac{\Gamma(m x+1)}{\Gamma(m x+\alpha+2)} .
\end{aligned}
$$


The series converges uniformly for $x \geqq \delta>0$ since $\alpha>\beta$. Replacing the binomial coefficient by $1 / m$ we obtain a majorant valid for the case $\beta=-1$. Thus in all cases $F(z)$ exists as a holomorphic function of $z$ for $x>0$, bounded for $x \geqq \delta>0$. In particular, it follows that the set $S\left(\alpha, \beta, \lambda_{n}\right)$ belongs to $L_{1}(0,1)$ since we can take $g(t)=1, z=\lambda_{n}$.

Now suppose the existence of a $g(t)$ such that for the corresponding function $F(z)$ we have $F\left(\lambda_{n}\right)=0,(n=1,2,3, \cdots)$, where the $\lambda_{n}$ satisfy conditions (i) and (ii). Since $F(z)$ is bounded and holomorphic in a half-plane, it must vanish identically by the well known analog of Blaschke's theorem. Moreover, we know that condition (ii) is necessary as well as sufficient for this conclusion as long as we are dealing with a bounded function whose zeros satisfy (i).

But $F(z)$ is the transform of the function $(1-t)^{\alpha} g(t)$ by the kernel $k_{\beta}\left(t^{z}\right)$. Thus the problem is reduced to the question of whether the vanishing of the transform implies the vanishing of the transformed almost everywhere. Or is it possible that such a transformation can admit of a non-trivial representation of zero? This question seems difficult to answer one way or the other if no restrictions are put upon the transformed function except the obvious requirement that the transform shall exist as an analytic function in some right half-plane. But we are limited to a particular class of functions, namely, those of the form $(1-t)^{\alpha} g(t)$, where $\alpha>\max (\beta,-1)$ and $g(t)$ is bounded, and for this class it turns out that the representations of zero are trivial.

Put

$$
t=e^{-u}, \quad k_{\beta}\left(t^{z}\right)=K_{\beta}(u z), \quad-(1-t)^{\alpha} g(t) \frac{d t}{d u}=G(u) .
$$

Then

$$
F(z)=\int_{0}^{\infty} K_{\beta}(u z) G(u) d u
$$

or

$$
F(z)=\sum_{m=1}^{\infty} m^{\beta} L(m z)
$$


Here $L(z)$ is the Laplace transform of $G(u)$, that is

$$
L(z)=\int_{0}^{\infty} e^{-u z} G(u) d u,
$$

or in terms of the old variables

$$
L(z)=\int_{0}^{1} t^{z}(1-t)^{\alpha} g(t) d t .
$$

The last formula shows that

$$
|L(z)| \leqq g \Gamma(\alpha+1) \frac{\Gamma(x+1)}{\Gamma(x+\alpha+2)}, \quad(x>0) .
$$

We can interpret (3) as a functional equation for the determination of $L(z)$ in terms of $F(z)$, where the solution is to satisfy (6). The solution is given by the special inversion formula of Möbius*

$$
L(z)=\sum_{n=1}^{\infty} \mu(n) n^{\beta} F(n z)
$$

where $\mu(n)$ is the factor of Möbius, that is,

$$
\mu(1)=1, \quad \mu(n)=0
$$

if $n$ has a quadratic factor, and

$$
\mu(n)=(-1)^{k}
$$

if $n$ is the product of $k$ distinct primes. In order to prove (7) rigorously, suppose that (3) is satisfied by a function $L(z)$, holomorphic in $x>0$, such that (6) holds for some choice of the constant $g$ and $\alpha>\max (\beta,-1)$. Then the double series

$$
\sum_{m=1}^{\infty} \sum_{n=1}^{\infty}(m n)^{\beta} L(m n z)
$$

is clearly absolutely convergent for $x>0$. Hence, if we multiply the equation

$$
\sum_{m=1}^{\infty} m^{\beta} L(m n z)=F(n z), \quad(n=1,2,3, \cdots)
$$

* A. F. Möbius, Journal für die reine und angewandte Mathematik, vol. 9 (1832), pp. 105-123, and Gesammelte Werke, vol. IV, pp. 591-612. 
by $\mu(n) n^{\beta}$ and add the results, the series so obtained is absolutely convergent, and can be rearranged at pleasure. Thus

$$
\begin{aligned}
\sum_{n=1}^{\infty} \mu(n) n^{\beta} F(n z) & =\sum_{n=1}^{\infty} \mu(n) \sum_{m=1}^{\infty}(n m)^{\beta} L(n m z) \\
& =\sum_{k=1}^{\infty} k^{\beta} L(k z) \sum_{d \mid k} \mu(d),
\end{aligned}
$$

where the last sum extends over the divisors of $k$. It is well known that this sum is zero unless $k=1$, when it equals one. Thus the double series reduces to a single term $L(z)$. This argument* proves that (7) gives the only solution $L(z)$ of (3) satisfying (6). In particular, if $F(z) \equiv 0$ and $L(z)$ satisfies (6), then $L(z) \equiv 0$. But this implies $G(u)=0$ almost everywhere, so that $g(t) \sim 0$. This completes the proof of Theorem 1 .

It should be remarked that if $F(z)$ is defined by (2), that is, $F(z)$ is the $K_{\beta}$-transform of a function $G(u)$ of the special form considered here, then $F(z)$ is also the unilateral Laplace transform of a function $H(u)$. We have

$$
\begin{aligned}
F(z) & =\int_{0}^{\infty} e^{-u z} H(u) d u, \\
H(u) & =\sum_{m=1}^{\infty} m^{\beta-1} G\left(\frac{u}{m}\right) .
\end{aligned}
$$

The last equation can also be regarded as a functional equation for the determination of $G(u)$ in terms of $H(u)$. Möbius' inversion formula applies, and gives

$$
G(u)=\sum_{n=1}^{\infty} \mu(n) n^{\beta-1} H\left(\frac{u}{n}\right)
$$

as the only solution of (10) which is $O\left(u^{\alpha}\right)$ for small values of $u$. In particular, if $H(u) \sim 0$, and $G(u)$ is known to be $O\left(u^{\alpha}\right)$, then $G(u) \sim 0$. Thus we have an automorphism between the theories of the two functional equations (3) and (10), as is to be expected a priori.

* See a similar argument by H. von Koch, Öfversigt af Kongliga Svenska Vetenskaps-Akademiens Förhandlingar, Stockholm, 1900, No. 5, pp. 659-668. Our functional equation could have been reduced to the system of linear equations in infinitely many unknowns treated by von Koch. 
3. Other Spaces. Similar results hold in $L_{p}(0,1), p>1$.

Theorem 2. The set $S\left(\alpha, \beta, \lambda_{n}\right)$ is complete in $L_{p}(0,1)$, $(1<p<\infty)$, if $\alpha>\max (\beta+1-1 / p,-1 / p)$, and $\Re\left(\lambda_{n}\right) \geqq \delta>0$, $\sum_{n=1}^{\infty} \Re\left(1 / \lambda_{n}\right)=+\infty$.

The proof follows the same lines as in the case $p=1$, the main difference being that $g(t)$ is now an arbitrary function in $L_{p^{\prime}}(0,1), 1 / p+1 / p^{\prime}=1$. The necessary estimates are obtained from Hölder's inequality. In particular, (6) has to be replaced by

$$
|L(z)| \leqq C\left[\frac{\Gamma(p x+1)}{\Gamma(p x+p \alpha+2)}\right]^{1 / p}, \quad(x>0),
$$

but the inversion formula (7) is still valid, and gives the only solution of (3) satisfying (12).

In order that $S\left(\alpha, \beta, \lambda_{n}\right)$ be in $C[0,1]$ it is necessary and sufficient that $\alpha \geqq \max (\beta+1,0), \Re\left(\lambda_{n}\right)>0$. But this set of functions can never be complete in $C[0,1]$ because they all vanish at $t=0$, and at $t=1$ if $\alpha>0$. This deficiency can be overcome by joining $f_{0}(t)=1$ to the set. In this way we get the following theorem.

Theorem 3. The set $1+S\left(\alpha, \beta, \lambda_{n}\right)$ is complete in $C[0,1]$ if $\alpha>\max (\beta+1,0), \Re\left(\lambda_{n}\right) \geqq \delta>0$, and $\sum_{n=1}^{\infty} \Re\left(1 / \lambda_{n}\right)=+\infty$.

By the theorem of F. Riesz we have to show that the only function $g(t)$ of bounded variation in $[0,1]$ such that

$$
\int_{0}^{1} f_{n}(t) d g(t)=0, \quad(n=0,1,2, \cdots),
$$

is a constant. We form

$$
F(z)=\int_{0}^{1} k_{\beta}\left(t^{z}\right)(1-t)^{\alpha} d g(t), \quad g(0)=g(1),
$$

where the end point condition expresses that (13) is satisfied for $n=0$. The estimates offer no particular difficulty, and lead to

$$
|L(z)| \leqq C \frac{\Gamma(x+1)}{\Gamma(x+\alpha+1)}, \quad(x>0) .
$$

Since $\alpha>\max (\beta+1,0)$, the inversion formula applies and gives the only solution $L(z)$ satisfying (14). In particular, if $F(z) \equiv 0$ and $L(z)$ satisfies $(14)$, then $L(z) \equiv 0$, whence 


$$
A(u) \equiv \int_{0}^{u}(1-t)^{\alpha} d g(t)=C,
$$

a constant, at all points of continuity of the integral. Integration by parts gives

$$
(1-u)^{\alpha} g(u)-g(0)+\alpha \int_{0}^{u}(1-t)^{\alpha-1} g(t) d t=C .
$$

The last term on the left is absolutely continuous, so $g(u)$ must be absolutely continuous in every interval $0 \leqq u \leqq 1-\delta$. But then by (15) we have $g^{\prime}(u) \sim 0$ and $g(u)=g(0)$ for $u<1$. Since $g(1)=g(0)$ by assumption, we have $g(u)=g(0), 0 \leqq u \leqq 1$. This completes the proof of Theorem 3 .

The only remaining case of interest in $C[0,1]$ is that in which $\alpha=\max (\beta+1,0)$. If $\beta<-1, \alpha=0$, we can still prove completeness of the set $1+S\left(\alpha, \beta, \lambda_{n}\right)$ if the $\lambda_{n}$ satisfy the conditions of Theorem 3. The case $\beta=-1, \alpha=0$, is excluded, but for $\beta>-1, \alpha=\beta+1$ our method fails. Take the simplest and most interesting case $\alpha=1, \beta=0$, that is, the set $S_{1}$ with unity adjoined. In order to prove the absolute convergence of the double series

$$
\sum_{m=1}^{\infty} \sum_{n=1}^{\infty} L(m n z)
$$

we obviously need something better than (14). Now it is not difficult to prove that

$$
\sum_{n=1}^{\infty}|L(n z)| \leqq \int_{0}^{1}(1-t) \frac{t^{x}}{1-t^{x}}|d g(t)|, \quad(x>0) .
$$

For fixed $x$ the multiplier of the integrator is a monotone increasing function of $t$, whence we conclude that the integral exists and is $O(1 / x)$. But this is clearly not enough for the convergence of the double series, so we do not know whether or not the inversion formula applies. It is perhaps of some interest to remark in this connection that the solution of the functional equation

$$
\sum_{n=1}^{\infty} L(n z)=\frac{1}{z} \quad \text { is } \quad L(z)=\frac{1}{z} \sum_{n=1}^{\infty} \frac{\mu(n)}{n}
$$

according to the inversion formula. By Landau's theorem the 
second series is identically zero, so the inversion formula does not give an actual solution. Under these circumstances we are forced to leave the question of the completeness of $S_{1}+1$ in $C[0,1]$ unanswered.*

Yale University and Massachusetts Institute of Technology

\section{GROUPS OF MOTIONS IN CONFORMALLY FLAT SPACES}

\section{BY JACK LEVINE}

1. Introduction. In this paper we consider the problem of determining the conditions which a conformally flat space must satisfy in order that it may admit a group of motions. These conditions are expressed in Theorem 1. Conformally flat spaces admitting simply transitive groups of motions are considered in the last section. All summations are from 1 through $n$ unless otherwise indicated.

2. Killing's Equations. The equations for determining the possible existence of groups of motions in a metric space are known as Killing's equations and are given by $\dagger$

$$
\xi^{k} \frac{\partial g_{i j}}{\partial x^{k}}+g_{i k} \frac{\partial \xi^{k}}{\partial x^{j}}+g_{j k} \frac{\partial \xi^{k}}{\partial x^{i}}=0 .
$$

If $V_{n}$ is conformally flat, there exists a coordinate system in which $g_{i j}=e_{i} \delta_{j}^{i} h^{2}$, where $e_{i}= \pm 1$. In this coordinate system (1) reduce to

$$
\begin{aligned}
e_{i} \frac{\partial \xi^{i}}{\partial x^{j}}+e_{j} \frac{\partial \xi^{j}}{\partial x^{i}} & =0, \quad(i \neq j, i, j \text { not summed }), \\
\xi^{k} \frac{\partial H}{\partial x^{k}}+\frac{\partial \xi^{i}}{\partial x^{i}} & =0, \quad(i \text { not summed, } H=\log h) .
\end{aligned}
$$

* The completeness of $1+S(\beta+1, \beta, \lambda)$ in $C[0,1]$ is proved for $-1<\beta \leqq 2$ in a paper to appear in the Annals of Mathematics.

$\dagger$ L. P. Eisenhart, Riemannian Geometry, p. 234. 